\title{
DOUBLE COMMUTANTS OF ALGEBRAIC OPERATORS ${ }^{1}$
}

\author{
T. ROLF TURNER
}

\begin{abstract}
The double commutant of an algebraic operator on a complex Hilbert space is aqual to the algebra (with identity) generated by that operator.
\end{abstract}

Introduction. Let $\mathfrak{A}$ be an algebra over some field, and let $\mathscr{S}$ be a subset of $\mathfrak{A}$. The commutant of $\mathscr{S}$ in $\mathfrak{A}$ is the set $\mathscr{S}^{\prime}$ of all elements of $\mathfrak{A}$ which commute with every element of $\mathscr{S}$. The double commutant of $\mathscr{S}$ in $\mathfrak{A}$ is the commutant of $\mathscr{S}^{\prime}$ in $\mathfrak{A}$. If $\mathscr{S}$ consists of a single element $s$, we shall speak of the commutant and double commutant of $s$ rather than of $\{s\}$.

Let $A$ be a linear transformation on a vector space. We say that $A$ is aigebraic if there exists a polynomial $p$ such that $p(A)=0$. In this paper we shall prove that if $\mathscr{H}$ is a complex Hilbert space, and $A$ belonging to $B(\mathscr{H})$ (the algebra of all bounded linear transformations on $\mathscr{H}$ ) is algebraic, then the double commutant of $A$ in $B(\mathscr{H})$ is equal to the algebra generated by $A$. This generalizes the well-known analogous result for finite-dimensional spaces (see [2, p. 113]), since any linear transformation on a finitedimensional vector space is algebraic.

It is already known (see [3 p. 72]) that if $T$ is an algebraic linear transformation on a vector space $\mathscr{V}$ then the double commutant of $T$ in $L(\mathscr{V})$ (the algebra of all linear transformations on $\mathscr{V}$ ) is equal to the algebra generated by $T$. This result does not imply our result for $B(\mathscr{H})$ however, since in general $L(\mathscr{H})$ is far larger than $B(\mathscr{H})$.

In the forthcoming the word operator will mean bounded linear transformation, and Hilbert space will mean complex Hilbert space.

1. Notation. If $A$ is an algebraic operator on a Hilbert space $\mathscr{H}$, we denote by $\mathfrak{U}_{A}$ the algebra (with identity) generated by $A$ in $B(\mathscr{H})$.

REMARK. In discussing operators on Hilbert space one often speaks of the weakly closed algebras which they generate. Since the algebra generated by an algebraic operator is finite dimensional it is closed in all of the usual

Recejved by the editors September 29, 1971.

AMS 1970 subject classifications. Primary 46L15, 47B99.

Key words anc phrases. Double commutant, algebraic, nilpotent, invariant subspace.

${ }^{1}$ This research constitutes part of the author's doctoral dissertation, written at the University of Michigan under the direction of Carl M. Pearcy.

(c) American Mathematical Society 1972 
topologies. Thus if $A$ is algebraic, $\mathfrak{U}_{A}$ is also the weakly closed algebra generated by $A$.

We prove our main result by dealing first with the special case of nilpotent operators. The crucial point in proving our theorem for nilpotent operators lies in showing that all of their invariant subspaces are $b i$ invariant (see [5]), that is, invariant under the double commutants of the operators.

We now state our theorem and the two lemmas upon which it depends.

THEOREM. If $A$ is an algebraic operator on a Hilbert space then $\mathfrak{A}_{A}=\mathfrak{U}_{\boldsymbol{A}}^{\prime \prime}$.

LEMMA 1. If $A$ is a nilpotent operator on a Hilbert space then every subspace invariant under $A$ is invariant under each element of $\mathfrak{I}_{A}^{\prime \prime}$.

LEMMA 2. If $A$ is a nilpotent operator on a Hilbert space then $\mathfrak{U}_{A}=\mathfrak{U}_{\boldsymbol{A}}^{\prime \prime}$.

Proof of Lemma 1. It clearly suffices to prove the lemma for cyclic invariant subspaces for $A$. (Every invariant subspace is spanned by cyclic invariant subspaces.) Let $e \in \mathscr{H}$ (the space on which $A$ acts) and suppose that $A^{n} e=0$ but $A^{n-1} e \neq 0$. Let $e_{1}=e, e_{2}=A e, \cdots, e_{n}=A^{n-1} e$, and let $\mathscr{H}_{1}$ be the subspace of $\mathscr{H}$ spanned by $e_{1}, \cdots, e_{n}$. (Notice that $\mathscr{H}_{1}$ is the cyclic invariant subspace for $A$ generated by $e$, and that $e_{1}, \cdots, e_{n}$ being linearly independent form a linear basis for $\mathscr{H}_{1}$.)

Let $\mathscr{H}_{2}=\mathscr{H}_{1}^{\perp}$, write $\mathscr{H}$ as $\mathscr{H}_{1} \odot \mathscr{H}_{2}$, and with respect to this decomposition write $A$ as a two-by-two operator matrix

$$
\left[\begin{array}{cc}
A_{1} & A_{2} \\
0 & A_{3}
\end{array}\right]
$$

Now let $D$ be in the double commutant of $A$, and write $D$ also as a two-bytwo operator matrix

$$
\left[\begin{array}{ll}
D_{1} & D_{2} \\
D_{3} & D_{4}
\end{array}\right]
$$

To show that $\mathscr{H}_{1}$ is invariant under $D$ it is necessary and sufficient to show that $D_{3}=0$.

Let $C$ be an operator from $\mathscr{H}_{2}$ to $\mathscr{H}_{1}$ such that $A_{1} C=C A_{3}$. Then the operator $\tilde{C}$ defined by

$$
\widetilde{C}=\left[\begin{array}{ll}
0 & C \\
0 & 0
\end{array}\right]
$$

is in the commutant of $A$, whence $C$ commutes with $D$. The equation $\widetilde{C} D=$ $D \tilde{C}$ tells us that

(1) $D_{3} C=0$ and

(2) $C D_{3}=0$. 
Our task is now to construct sufficiently many operators $C$ so that these two equations force $D_{3}$ to be zero.

The fact that $A$ is nilpotent implies that $A_{3}$ is nilpotent also. Let the order of nilpotence of $A_{3}$ be $k$. We consider two cases.

Case I. $k \geqq n$. (Recall that $n$ is the order of nilpotence of $A_{1}$.) The order of nilpotence of $A_{3}$ is also that of $A_{3}^{*}$. Since $k \geqq n$, we may find $g$ in $\mathscr{H}_{2}$ such that $A_{3}^{* n} g=0$ and $A_{3}^{* n-1} g \neq 0$. Let

$$
\begin{aligned}
& g_{1}=A_{3}^{* n-1} g, \\
& g_{2}=A_{3}^{* n-2} g, \\
& \cdot \\
& \cdot \\
& \cdot \\
& g_{n}=g .
\end{aligned}
$$

Define $C: \mathscr{H}_{2} \rightarrow \mathscr{H}_{1}$ by

$$
C f=\left(f, g_{1}\right) e_{1}+\cdots+\left(f, g_{n}\right) e_{n} .
$$

Then it is clear that $C$ is an operator, that $A_{1} C=C A_{3}$, and that Range $C$ is equal to $\mathscr{H}_{1}$. Consequently, for any $h$ in $\mathscr{H}_{1}, h=C f$ for some $f$ in $\mathscr{H}_{2}$. Therefore $D_{3} h=D_{3} C f=0$ (by equation (1)). Hence $D_{3}=0$.

Case II. $k<n$. Let $g$ be any nonzero element of $\mathscr{H}_{2}$, and let $r$ be the first positive integer such that $A_{3}^{* r} g=0$. Note that $r \leqq k<n$. Let $m=n-r$. Let

$$
\begin{aligned}
& g_{1}=A_{3}^{* r-1} g, \\
& g_{2}=A_{3}^{* r-2} g, \\
& \cdot \\
& \cdot \\
& \cdot \\
& g_{r}=g .
\end{aligned}
$$

Define $C: \mathscr{H}_{2} \rightarrow \mathscr{H}_{1}$ by

$$
C f=\left(f, g_{1}\right) e_{m+1}+\cdots+\left(f, g_{r}\right) e_{n} .
$$

Again $A_{1} C=C A_{3}$. Note that $C g \neq 0$. Thus for each nonzero $g$ in $\mathscr{H}_{2}$ there exists $C: \mathscr{H}_{2} \rightarrow \mathscr{H}_{1}$ such that $A_{1} C=C A_{3}$ and $g \notin \operatorname{Ker} C$. In other words $\bigcap_{A_{1} C=C A_{3}} \operatorname{Ker} C=\{0\}$. Now for any $h$ in $\mathscr{H}_{1}$ and any $C$ such that $A_{1} C=$ $C A_{3}, C D_{3} h=0$ (by equation (2)). Thus $D_{3} h$ is in $\operatorname{Ker} C$ for all such $C$, that is, $D_{3} h \in \bigcap_{A_{1} C=C A_{3}} \operatorname{Ker} C=\{0\}$. Consequently $D_{3} h=0$ for each $h$ in $\mathscr{H}_{1}$ so $D_{3}=0$.

Proof of LemMa 2. Our technique is essentially that used to prove the von Neumann Double Commutant Theorem (see [1, p. 40]).

Let $\mathscr{H}$ be the sprace on which $A$ acts. 
For an arbitrary operator $T$ on $\mathscr{H}$ and arbitrary $n$, let $T^{(n)}$ denote the direct sum of $T$ with itself $n$ times. The operator $T^{(n)}$ acts on the space $\mathscr{H}^{(n)}$, the direct sum of $\mathscr{H}$ with itself $n$ times. Note that if $T$ is nilpotent, then $T^{(n)}$ is nilpotent for any $n$. Let $D$ be in the double commutant of $A$ and let $\mathscr{N}=\left\{X \mid\left\|(X-D) f_{i}\right\|<1, i=1, \cdots, k\right\}, f_{1}, \cdots, f_{k} \in \mathscr{H}$, be a basic strong neighborhood of $D$. The operator $D^{(k)}$ is clearly in the double commutant of $A^{(k)}$, and since $A^{(k)}$ is nilpotent, every subspace of $\mathscr{H}^{(k)}$ invariant under $A^{(k)}$ is also invariant under $D^{(k)}$ by Lemma 1 . In particular the cyclic invariant subspace $\mathscr{M}$ generated by $f=\left(f_{1}, \cdots, f_{k}\right)$ is invariant under $D^{(k)}$. Thus $D^{(k)} f \in \mathscr{M}$ and we conclude that there is a polynomial $p$ such that $\left\|\left(p\left(A^{(k)}\right)-D^{(k)}\right) f\right\|<1$. Therefore $\left\|(p(A)-D) f_{i}\right\|<1$ for $i=1, \cdots, k$, and so $p(A) \in \mathcal{N}$. We conclude that every strong neighborhood of $D$ contains a polynomial in $A$, so that $D$ is in the strong closure of the algebra generated by $A$, which is just the algebra itself.

Proof OF THEOREM. Let $p$ be the minimal polynomial of $A$ and factor $p$ as $\left(z-v_{1}\right)^{n_{1}} \times \cdots \times\left(z-v_{r}\right)^{n_{r}}$, where $v_{1}, \cdots, v_{r}$ are the distinct roots of $p$. Let

$$
\mathscr{M}_{i}=\operatorname{Ker}\left[\left(A-v_{1}\right)^{n_{1}} \times \cdots \times\left(A-v_{i}\right)^{n_{i}}\right],
$$

for $i=1, \cdots, r$. Let $\mathscr{H}_{1}=\mathscr{M}_{1}$, and $\mathscr{H}_{i}=\mathscr{M}_{i} \cap \mathscr{M}_{i-1}^{\perp}$, for $i=2, \cdots, r$. Then $\mathscr{H}$ may be written as the orthogonal direct sum $\mathscr{H}_{1} \oplus \cdots \oplus \mathscr{H}_{r}$.

With respect to this direct sum decomposition we may write $A$ as an $r$-by- $r$ operator matrix which is upper triangular and whose $i$ th diagonal entry is of the form $B_{i}+v_{i}$, where $B_{i}$ is nilpotent of order $n_{i}$, for $i=1, \cdots$, $r$. Since the $v_{i}$ are distinct, $A$ is therefore similar to the operator $B=$ $\left(B_{1}+v_{1}\right) \oplus \cdots \oplus\left(B_{r}+v_{r}\right)$.

(To see this last assertion, proceed by induction and apply Rosenblum's theorem that if the spectra of $P$ and $Q$ are disjoint, then the operator equation $P X-X Q=R$ has a solution in $X$ for any $R$ [4].)

Again applying the fact that $\nu_{1}, \cdots, v_{r}$ are distinct we observe that the algebra generated by $B$ is the direct sum of the algebras generated by the $B_{i}+v_{i}$. For each $i=1, \cdots, r$, the algebra generated by $B_{i}$ is equal to its double commutant by Lemma 2. The same is therefore true of the operators $B_{i}+v_{i}$. It now follows that $\mathfrak{A}_{B}=\mathfrak{U}_{B}^{\prime \prime}$, and since $A$ is similar to $B$, that $\mathfrak{U}_{A}=\mathfrak{U}_{A}^{\prime \prime}$.

REMARK. It has recently come to the author's attention that J. A. Deddens has obtained the same result, using different methods.

\section{BIBLIOGRAPHY}

1. J. Dixmier, Les algèbres d'opérateurs dans l'espace hilbertien, Gauthier-Villars, Paris, 1969.

2. N. Jacobson, Lectures in abstract algebra. Vol. II. Linear algebra, Van Nostrand, Princeton, N.J., 1953. MR 14, 837. 
3. I. Kaplansky, Infinite abelian groups, rev. ed., Univ. of Michigan Press, Ann Arbor, Mich., 1969. MR 38 \#2208.

4. M. Rosenblum, On the operator equation $B X-X A=Q$, Duke Math. J. 23 (1956), 263-269. MR 18, 54.

5. N. Salinas and D. Herrero, Analytically invariant and bi-variant subspaces (to appear).

Department of Mathematics, University of Alberta, Edmonton, Alberta, Canada 